\title{
A SOHO/UVCS study of coronal outflows at the edge of an active region complex
}

\author{
L. Zangrilli ${ }^{1}$ and G. Poletto ${ }^{2}$ \\ ${ }^{1}$ Istituto Nazionale di Astrofisica (INAF) - Osservatorio Astrofisico di Torino, Strada Osservatorio 20, 10025 Pino Torinese, Italy \\ e-mail: zangrilli@oato.inaf.it \\ 2 Istituto Nazionale di Astrofisica (INAF) - Osservatorio Astrofisico di Arcetri, Largo Enrico Fermi 5, 50125 Firenze, Italy
}

Received 21 April 2012 / Accepted 14 June 2012

\section{ABSTRACT}

\begin{abstract}
Context. In the past, active regions (ARs) have been suggested as a possible source of the slow solar wind. Their role as solar wind contributors has recently been supported by HINODE observations at low coronal levels.

Aims. Our purpose is to determine whether outflows at the edges of ARs can be detected in higher layers of the corona, supporting the low-corona evidence for the occurrence of wind streams from ARs.

Methods. Data acquired by SOHO/UVCS on January 2, 1998, at altitudes ranging from 1.5 to 2.3 solar radii at mid latitudes, were used to infer the physical properties of an AR complex at the time of its limb passage. To this end, the Doppler dimming technique was applied to UVCS observations of the $\mathrm{H}_{\mathrm{I}} \mathrm{Ly} \alpha$ and O vi doublet lines at 1031.9 and $1037.6 \AA$.

Results. Outflow speeds (and electron densities) were inferred: outflows, at speeds increasing with height, turn out to be confined within a narrow channel at the edge of closed loop systems within the AR. Our results are compared with those obtained by other authors with different techniques.

Conclusions. Our results support the assumption that ARs are sources of slow wind. To our knowledge these are the first direct measurements of AR flows in the intermediate corona. Tentative profiles of the speed vs. heliocentric altitudes at heliocentric distances between $\approx 1.5$ and $\approx 2.3$ solar radii show that AR flows are faster than streams from equatorial coronal holes.
\end{abstract}

Key words. solar wind - Sun: UV radiation - Sun: activity

\section{Introduction}

The source of the fast solar wind has been successfully identified when, about 40 years ago, Krieger et al. (1973) examined the soft X-ray images acquired in 1970 during a sounding rocket flight, and claimed that a coronal hole, covering a large area of the Sun, was responsible for the recurrent fast wind streams observed in situ. On the other hand, sources of the slow wind have not been unequivocally identified yet, although quite a good number of candidates have been suggested, from streamer cusps to equatorial holes, coronal hole $(\mathrm{CH})$ boundaries and active regions (ARs). Identifying the solar wind sources implies either detecting plasma flows in the chromosphere/corona via remote observations, or measuring the solar wind speed with in situ experiments and tracing back the observed flows to the regions at which they originated.

In the following, we focus on the role possibly played by ARs as contributors to the solar wind. Kojima et al. (1999) reconstructed with a tomographic scintillation analysis the solar wind distribution on a source surface set, as usual, at $2.5 R_{\odot}$, and concluded that a slow-speed stream was emanating from one side of an active region. However, in situ evidence for the AR origin of a solar wind component may be challenged on the basis of the uncertainties in the mapping-back procedures (usually a two-step technique involving a ballistic plus a potential magnetic field extrapolation). On the other hand, there is another factor that helps establishing the wind-AR association, namely, a comparison of the in situ slow-wind abundances vs. the coronal abundances of the AR that are predicted to be the source of the observed slow wind. This attempt has been made recently by Brooks \& Warren (2011), who calculated the $\mathrm{Si} / \mathrm{S}$ abundance ratio in an AR observed by HINODE/EIS over a five-day interval and found it to be consistent with the low first ionization potential (FIP) element enhancement measured a few days later by the ACE/SWICS experiment. An analogous study has been performed by Liewer et al. (2004), who pointed out from an analysis of ACE and Ulysses data, that the $\mathrm{O}^{7+} / \mathrm{O}^{6+}$ ratio (a proxy for the temperature of the region where freezing-in occurs) is in the solar wind streams, which purportedly originate from ARs, higher and more variable than the fast wind ratio.

The other approach to the wind source identification, based on the analysis of remotely acquired coronal data, has been flourishing over recent years because of the availability of new data from space experiments. About ten years ago, Winebarger et al. (2001) measured continuous intermittent outflows in a corner of an AR in TRACE data, but did not make any reference to a possible contribution to solar wind. Subsequently, HINODE data provided a wealth of measurements that seem to confirm the presence of outflows in ARs and a direct association with solar wind was explicitly made. Sakao et al. (2007) identified a pattern of continuously outflowing plasma originating at the edge of an AR that was observed over three days by HINODE/XRT, and claimed this to be the first direct observation of outflowing material from ARs. XRT results were also supported by HINODE/EIS Doppler measurements. A crude estimate showed that per unit time, the AR flows might contribute as much as $1 / 4$ th of the total mass loss rate of the solar wind.

The work of Sakao and collaborators has been followed by others, who analyzed the properties of AR outflows, trying to 
evaluate the outflowing plasma speed and to establish how frequently outflows occur. Harra et al. (2008) measured continuous Doppler shifts that ranged between 20 and $50 \mathrm{~km} \mathrm{~s}^{-1}$, from HINODE/EIS spectra, from which the authors estimated an outflow speed of possibly $100 \mathrm{~km} \mathrm{~s}^{-1}$. Unresolved high-speed upflows have been suggested by Doschek et al. (2008) still from the analysis of line profiles in HINODE/EIS spectra, while Guo et al. (2010) claimed from XRT data that persistent outflows, with projected flow speeds higher than $200 \mathrm{~km} \mathrm{~s}^{-1}$, often occur intermittently, with a period of a few minutes. From HINODE and TRACE data He et al. (2010) analogously inferred speeds occasionally exceeding the coronal sound speed, even at very low altitudes $(\leq 100 \mathrm{Mm})$, in outflows recurring about every $20 \mathrm{~min}$. However, sporadic downflows in ARs have been detected in EIS data by Ugarte-Urra \& Warren (2011) and by Kamio et al. (2011) in the SDO/AIA experiment.

As the characteristics of flows at AR boundaries are being defined, we point out that all remotely acquired data refer to low coronal levels, while, obviously, in situ data refer to quite large heliocentric distances. The paper of Ko et al. (2006) analyzed data acquired over a one-week time interval by SOHO/UVCS at $1.64 R_{\odot}$, in the intermediate corona. At that time, an equatorial $\mathrm{CH}$, followed by an AR complex, was crossing the west limb of the Sun. Ko et al. (2006) compared abundances inferred from UVCS spectra with abundances measured in situ by ACE and concluded that some slow wind originated in the boundary region between the $\mathrm{CH}$ and the AR. However, we do not have any estimate of the flow speed in this altitude range and, because the origin and the role played by the flows is not completely clear, this information may be crucial for a better interpretation of the data. The present work aims at filling this gap by presenting results inferred from data acquired by SOHO/UVCS at altitudes ranging from 1.5 to $2.3 R_{\odot}$. It is well known that the Doppler dimming technique allows one to infer outflow speeds from $\mathrm{H}_{\mathrm{I}}$ and $\mathrm{O}$ vi spectral data: in the following we give the outflows inferred from UVCS at the edges of an AR complex observed at its limb transit in 1998, two years after solar minimum, as a function of the heliocentric altitudes.

The paper is organized as follows: in the next section we describe the data; then we introduce the procedure followed to infer densities and outflow speeds in the examined region and our results. These are discussed in the context of previous works at the end of the paper.

\section{The data}

To ascertain whether flows occur at the AR edges, we need to find observations of an isolated AR, at the time it crosses the solar limb, so that the distribution of flows, if any, around the AR would be clearly identifiable. To this end, we selected AR NOAA 8124 which on January 2, 1998, was the only bright feature at the limb of the Sun, at south 21, west 91 (see Fig. 1, first panel). This AR complex was first identified on October 28, 1997, at S21, E67 and showed a beta-type configuration and a negative leading polarity. Here we examine its third limb passage when it appeared as a medium-size bipolar region (see Green et al. 2001; Green et al. 2002).

On January 2, SOHO/UVCS (see e.g. Domingo et al. 1995; Kohl et al. 1995; Kohl et al. 1997) made synoptic observations of the Sun from $\approx 05$ UT to $\approx 09$ UT. Synoptic data are taken at eight different polar angles, every 45 degrees, starting from the North Pole, with the UVCS slit normal to the radial of the Sun, at heliocentric distances that at the equator range from 1.5 to 3.5 solar radii and reach lower heights in lower latitudes scans.
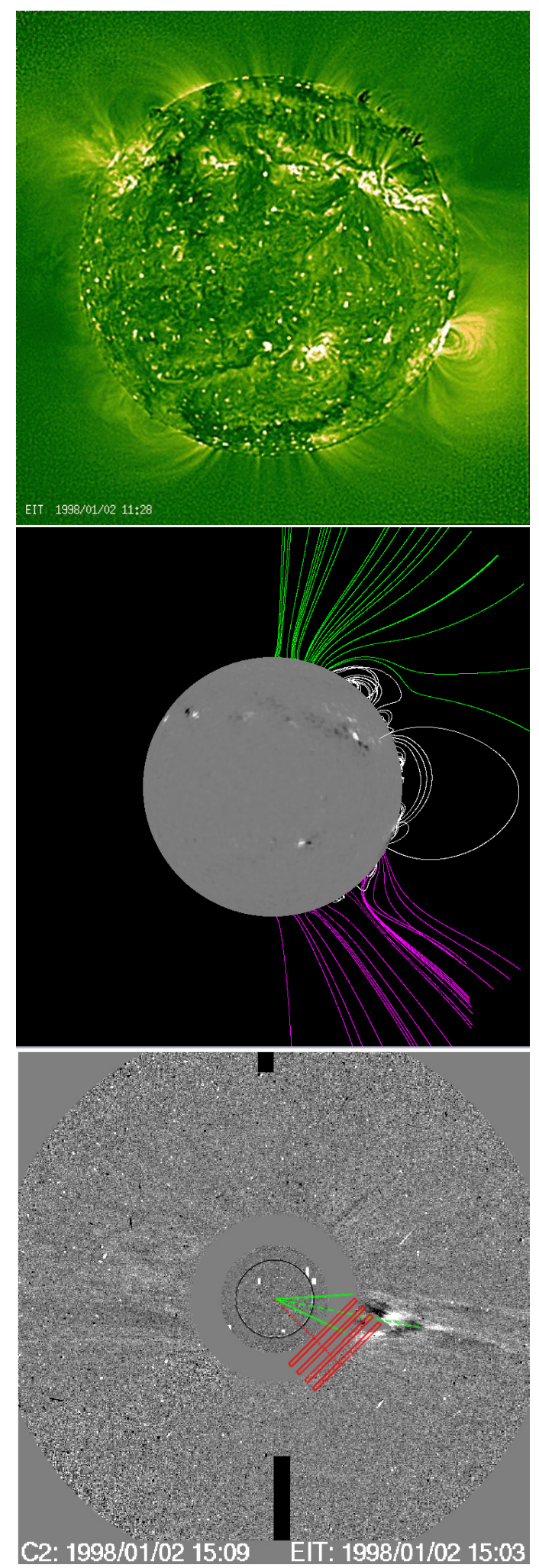

Fig. 1. First panel: EIT $195 \AA$ Aavelet-enhanced image of the Sun on January 2, 1998, when AR 8124 crossed the southwest limb of the Sun. Second panel: potential field extrapolation on January 2, 1998, in the western hemisphere, showing closed/open fieldlines at the northern/southern edge of the AR, respectively. Third panel: LASCO C2 difference image at 15:09 UT showing the propagating disturbance that originated at 14:30 UT at PA $=259$. The black circle indicates the limb of the solar disk. The sun-centered cone shows the CME width and axis at 14:30 UT, as given in the LASCO CME catalog. The four slits of UVCS observations we analyzed are also shown, together with the radial at 225 degrees. 


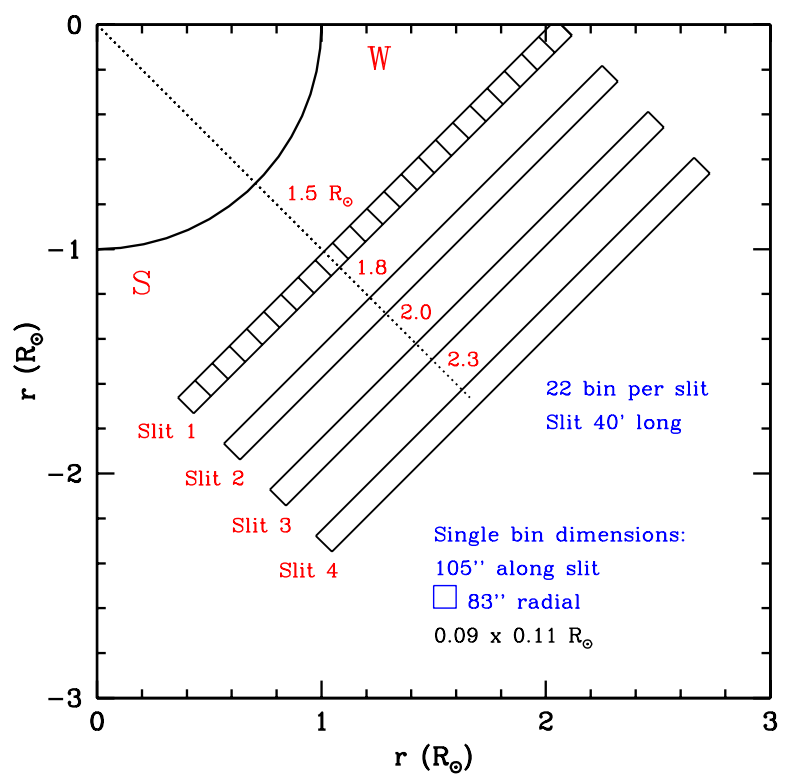

Fig. 2. Cartoon illustrating the positions of the UVCS slit and other properties of the data acquired on January 2, 1998.

Data covering AR 8124 were acquired both in the equatorial and the southwest mid-latitude scan: we used the latter, because potential field extrapolations show only closed fieldlines at the northern edge of the AR (see Fig. 1, second panel).

UVCS observations at PA $=225$ degrees (southwest direction) were taken both in the $\mathrm{H}_{\mathrm{I}} \mathrm{Ly} \alpha$ and the $\mathrm{O}$ VI channel at the four heliocentric distances $1.5,1.8,2.1,2.3 R_{\odot}$ (slit positions $1,2,3,4$ in the following). The instantaneous field of view is $40^{\prime}$ along the slit direction. In the radial direction, the $\mathrm{O}$ vi channel slit width was $0.3 \mathrm{~mm}$ wide, corresponding to $83^{\prime \prime}$ in the plane of the sky, while the $\mathrm{H}_{\mathrm{I}} \mathrm{Ly} \alpha$ channel slit width was $50 \mu \mathrm{m}$ wide, corresponding to $14^{\prime \prime}$. Data were acquired with a spatial resolution of $21^{\prime \prime}$ per bin: an additional summation over 5 bins was performed when analyzing the data to improve the signal-to-noise ratio. Hence, we obtained 22 points along the slit and a spatial resolution of 105" in the direction normal to the radius of the Sun. The spatial bin coordinates increase along the southward direction. In the following, each data point in our analysis is representative of an area $0.09 \times 0.11 R_{\odot}$ wide, in the $\mathrm{O}$ VI and $0.015 \times 0.11 R_{\odot}$ in the Hi Ly $\alpha$ channels, respectively. The spectral bandwidth of O vi data is $0.594 \AA$ per bin, because a spectral binning over 6 pixels per bin is usually adopted in synoptic observations. In the $\mathrm{H}_{\mathrm{I}} \mathrm{Ly} \alpha$ channel the spectral bandwidth is $0.14 \AA$ per bin.

A cartoon illustrating the main characteristics of our data set appears in Fig. 2, showing the field of view covered by the observations and the data points along the four slits for the $\mathrm{O}$ vi channel. The case of $\mathrm{H}_{\mathrm{I}} \mathrm{Ly} \alpha$ data is similar, except for the slit width, which covered a coronal area six times smaller along the radial direction.

The spectrum acquired in the $\mathrm{O}$ VI channel includes weaker lines in addition to the $\mathrm{O}$ vi doublet lines, such as the Si XII lines at $499.4 / 520.7 \AA$, detected in the second order, and the H I Ly $\beta$ at $1025.7 \AA$. Exposure times were increased as a function of the slit altitude, and range from $300 \mathrm{~s}$ at $1.5 R_{\odot}$ solar radii to 900 at the highest position of the slit.

The UVCS Data Analysis Software (DAS) was used to derive the observed line intensities by integrating fitted Gaussian profiles after wavelength and radiometric calibration.
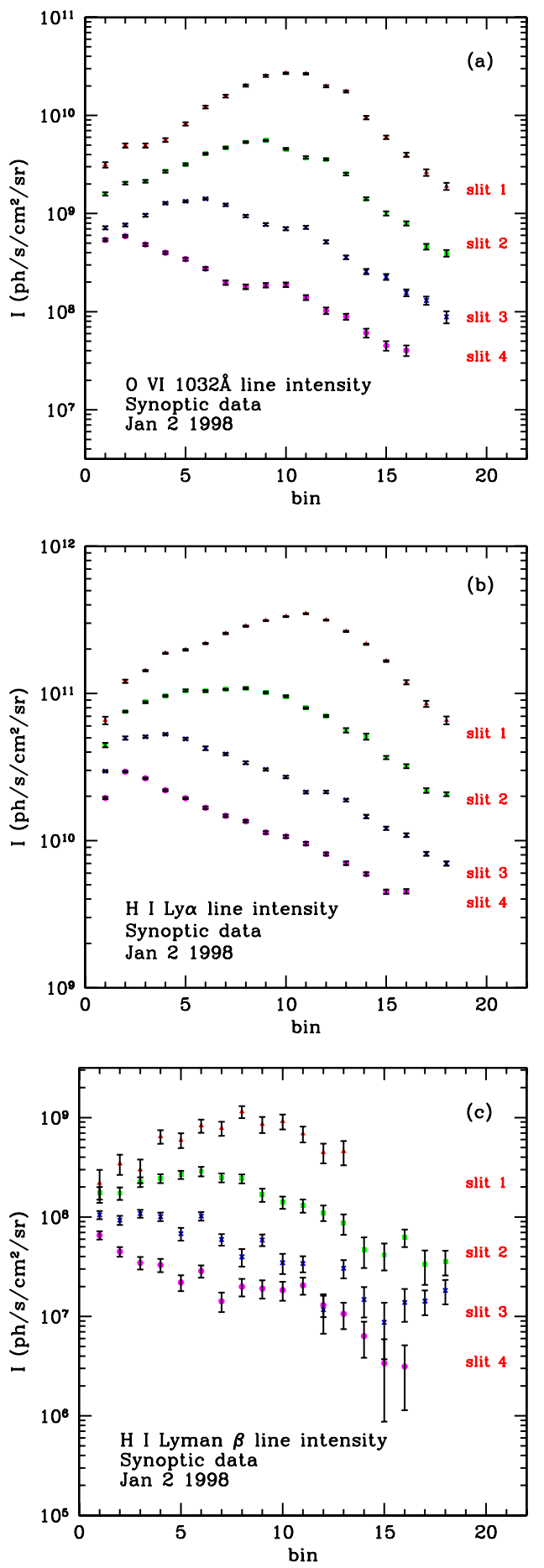

Fig. 3. Panel a) O vi $1031.9 \AA$ line intensities along the UVCS slit at the four altitudes where the UVCS slit was set. The spatial bin values increase along the southward direction. We show only data in bins 1 to 18 for the first three slits and from 1 to 16 for the fourth. Panel b) same as panel a) for the $\mathrm{H}_{\mathrm{I}} \mathrm{Ly} \alpha$ line. Panel c) same as panel a) for the $\mathrm{H}_{\text {I Ly }} \beta$ line, except in the first slit, where data were considered only up to bin 13 . Intensities are given in $\mathrm{ph} / \mathrm{s} / \mathrm{cm}^{2} / \mathrm{sr}$.

We corrected for the stray light following the semi-empirical relationships given by Cranmer et al. (2010).

Figure 3 gives intensities of the Ovi 1031.9 (panel a), HI Ly $\alpha$ (panel b) and Hi Ly $\beta$ (panel c) lines in bins 1 through 18 for slits $1-3$, and bins 1 through 16 for slit 4 , because the statistics of the other data points is too poor. $\mathrm{H}_{\text {I }} \mathrm{Ly} \beta$ data relative to 


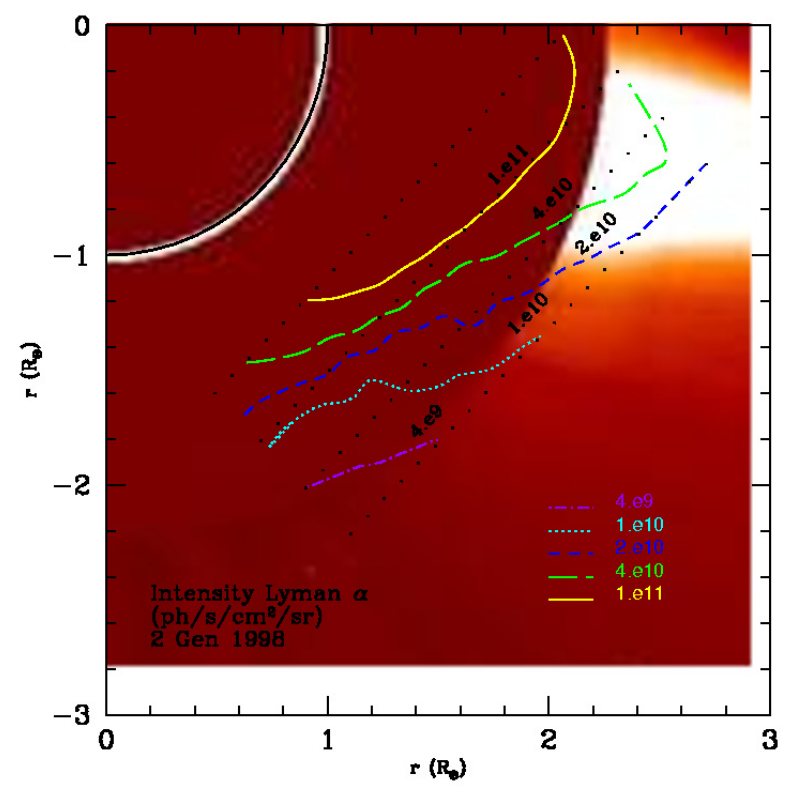

Fig. 4. White-light corona as seen by SOHO/LASCO $\mathrm{C} 2$ on January 2, 1998, and superimposed isocontours of the $\mathrm{H}_{\mathrm{I}} \mathrm{Ly} \alpha$ intensity from $\mathrm{SOHO} / \mathrm{UVCS}$. The streamer axis is at $\mathrm{PA} \approx 11$ degrees south of the equator.

the first slit were considered only up to bin 13 owing to the low signal-to-noise ratios of the corresponding data. Intensities were derived by fitting the observed spectrum within selected wavelength intervals with a least-squares Gaussian line fit, allowing us to solve ambiguities caused by blended line wings, which are particularly obvious in the $\mathrm{O}$ vi doublet, and to better determine the background level. Error bars in Fig. 3 give the $\pm 1 \sigma$ standard deviation of the Poisson photon counting statistics.

A comparison between the visible light corona as seen by $\mathrm{SOHO} / \mathrm{LASCO} \mathrm{C} 2$ and the corona in the $\mathrm{H}_{\mathrm{I}} \mathrm{Ly} \alpha$ line as seen by UVCS is shown in Fig. 4. The H I Ly $\alpha$ isophotes were calculated with a spline interpolation of the measured intensities along the slits. The streamer structure is clearly visible, its axis is about 11 degrees below the equator in the western hemisphere.

In Fig. 5 the profile along the slit of the 1031.9 to $1037.6 \mathrm{O}$ vi lines intensity ratio is given at the four altitudes where the slit was set. As we show in Appendix A, the ratio is independent of the oxygen abundance and is considered to be a proxy for the outflow speed of the plasma (see e.g. Noci et al. 1987). We will discuss the figure below in more detail.

We now examine whether there was any activity in the AR on January 2, 1998, that might modify the area in and around the AR itself. In particular, we are interested in coronal mass ejections that might promote transient plasma outflows, while passing through the UVCS slit. On January 2, there were only three CMEs, two of them being irrelevant to us: the first, at 4:37 UT occurred before we started taking data and was originating in the northern hemisphere; the second, a halo CME, started after our data were acquired. At 14:30 UT a CME described as "a small linear disturbance" traveling along the western streamer might potentially have affected our data. However, LASCO C2 difference images (see Fig. 1, third panel) clearly show that the event, centered at PA $=259$ degrees with a width of 29 degrees, occurred far away from the potential site of outflows, as indicated by the potential field extrapolations. We conclude that there was no activity in AR 8124 that might give rise to flows that could interfere with our study.

\section{Determining electron densities and outflow speed}

Usually, when temperatures and electron densities are known, outflow speeds are evaluated by a Doppler dimming analysis of the $\mathrm{O}$ vi doublet lines: because these lines form through collisional and radiative excitation, their resonantly scattered component can be reduced by the Doppler dimming effect. Therefore its value is higher in a static atmosphere than in an outflowing plasma. By evaluating the amount by which the radiative component is reduced, the outflow speed of the plasma can be deduced. This technique has been adopted by e.g. Cranmer et al. (1999) to build an empirical model of a coronal hole at solar minimum, using densities inferred from white-light data.

In the present case, we did not have an independent means to derive densities in the region we analyzed, because white-light polarized brightness, $\mathrm{pB}$, measured by $\mathrm{Mk} 3$ and LASCO C2 coronagraphs does not cover the whole latitude and the whole altitude interval we examined. Hence we adopted a different technique than used by Cranmer et al. (1999), which allows us to evaluate simultaneously densities and outflow speeds via an iterative procedure that has been devised by Zangrilli et al. (2002), when building a 2D model of a coronal hole. Here we briefly summarize this procedure; we refer the reader to the 2002 paper for more details.

In an outflowing atmosphere, the total intensities of the 1031.9 and 1037.6 $\AA \mathrm{O}$ vi doublet lines can be expressed by

$I_{1031.9}=2 C\left(n_{\mathrm{e}}\right)+4 \mathcal{R}\left(n_{\mathrm{e}}, v_{\mathrm{OVI}}\right)$
$I_{1037.6}=\mathcal{C}\left(n_{\mathrm{e}}\right)+\mathcal{R}\left(n_{\mathrm{e}}, v_{\mathrm{OVI}}\right)+\mathcal{P}\left(n_{\mathrm{e}}, v_{\mathrm{OVI}}\right)$,

where $C$ and $\mathcal{R}$ are the collisional and radiative components of the Ovi $\lambda 1037.6 \AA$ line, whose values are half and 0.25 of the analogous components of the 1031.9 line, respectively. $\mathcal{P}$ is a term that takes into account the exciting effect of the $\mathrm{C}$ II chromospheric radiation: for sufficiently high outflows, the intensity of the Ovi $\lambda 1037.6 \AA$ line increases because its radiative component is excited by the chromospheric $\mathrm{C}_{\text {II }}$ lines at 1036.3 and $1037.0 \AA$. This phenomenon is known as pumping effect.

For a more detailed discussion about the formation of the $\mathrm{O}$ vi doublet lines in the corona we refer to Appendix A. We point out two relevant issues before proceeding: a) it is easily seen from Eqs. (A.3), (A.2) and (A.4) that the emissivity of the collisional component depends on $n_{\mathrm{e}}^{2}$, while the emissivity of the resonantly scattered component depends on $n_{\mathrm{e}}$. As a consequence, the radiative component can be detected farther out from the Sun than the collisional component and outflows can be inferred at large distances. Moreover, b) from Eqs. (A.4) and (A.2) we realize that the ratio of the 1031.9 to the $1037.6 \AA$ line emissivity is independent of the element abundance and the ionization balance. Hence, uncertainties in these parameters do not affect the outflow speed determination.

The line intensities in the above equations strongly depend on the electron density and the outflow speed (and of course on the ion abundance), which are the unknown physical quantities to be found (as fully described in the next section, intensities depend, although more weakly, on other parameters as well). We searched for a solution of the system (1) through an iterative numerical procedure, initiated by assuming a first-guess value for density and speed, calculating $C, \mathcal{R}$ and $\mathcal{P}$, and making a comparison with the observed line intensities. Then the values for density and speed were properly adjusted, and the cycle was restarted until the convergence between calculated and measured 

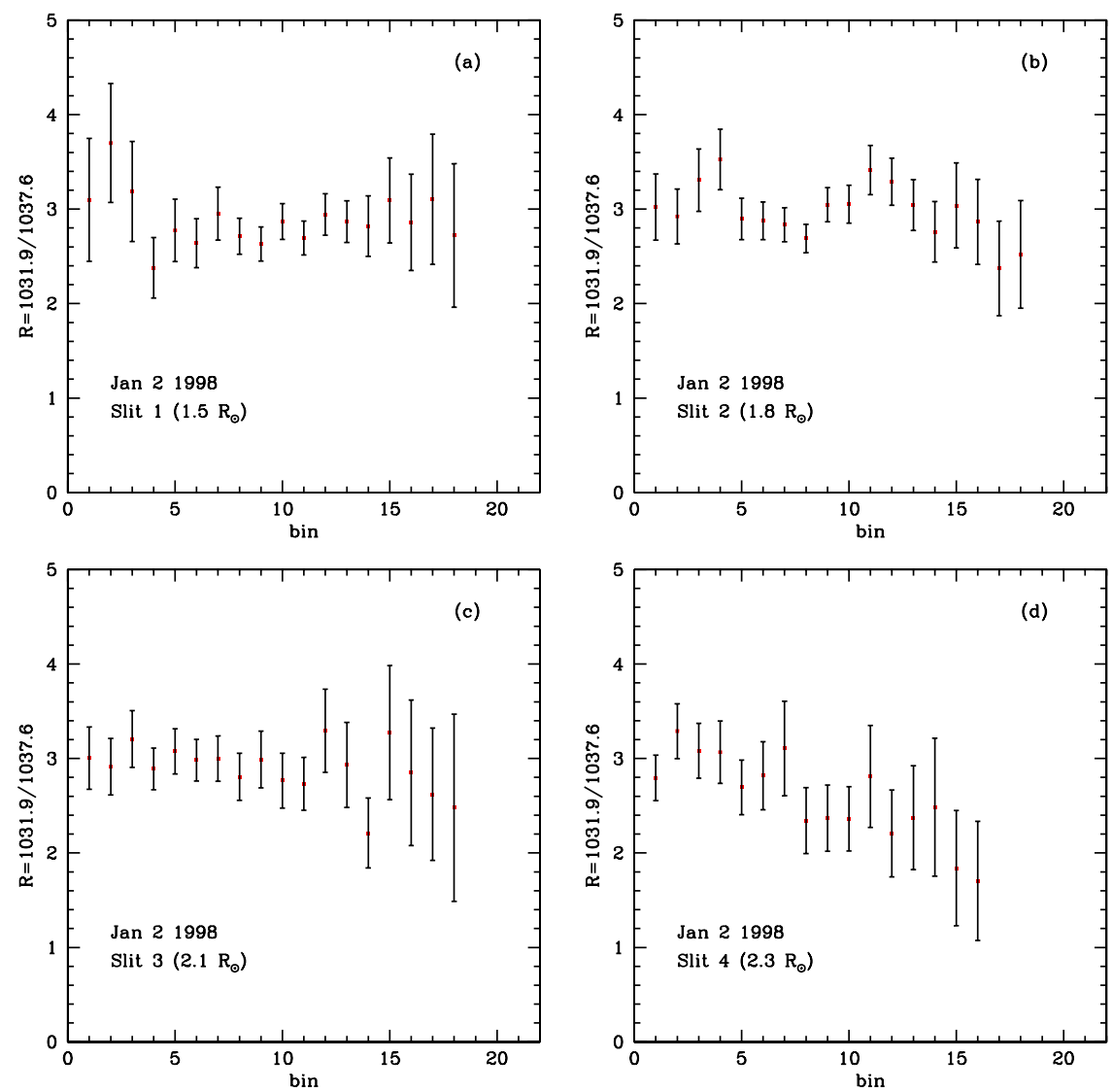

Fig. 5. Ratio of the 1031.9 to 1037.6 $\mathrm{O}$ vi line intensities as a function of the bin coordinate: panels a)-d) correspond to slit $1,2,3,4$ respectively.

intensities was reached. The procedure for the solution distinguishes two cases of low-speed and high-speed regime. A lowspeed regime $\mathcal{P}$, and a high-speed regime $\mathcal{R}$, are considered as correction terms, respectively, and therefore their initial values are assumed to be zero, and are updated at the end of each cycle of the iteration.

We refer the reader to Zangrilli et al. (2002) for a discussion of the convergence and uniqueness of the solution of the system with the method that we briefly summarized. In the next section we give the full expression of $\mathcal{P}$ and $\mathcal{R}$ to show all parameters we need to calculate the $\mathrm{O}$ vi line intensities.

\subsection{Results}

\subsubsection{Model parameters}

We have mentioned that to synthesize the line intensities and derive electron densities and outflow speeds, we need to know several other parameters, such as the profiles vs. heliocentric distances of the electron temperature, of the parallel and of the perpendicular kinetic temperatures, and the value of the disk intensity and the element abundance. Knowledge of the parallel and kinetic (perpendicular) temperatures allows us to define the plasma anisotropy and use the method described in the previous subsection, with appropriate values of the perpendicular to parallel temperature ratio. However, there is no means to evaluate the parallel temperature, and, as far as the perpendicular temperature is concerned, synoptic data from the $\mathrm{O}$ vi channel are taken with too wide a slit to yield a proper evaluation of kinetic temperatures. In contrast, $\mathrm{H}_{\mathrm{I}} \mathrm{Ly} \alpha$ data were acquired with a narrower slit and a higher spectral resolution binning, therefore it is possible to obtain the local kinetic temperature of protons from the analysis of the H I Ly $\alpha$ spectral line width. Furthermore, protons and oxygen ion speed may be different, as is known to occur in coronal holes (see, e.g. Cranmer et al. 1999). We conclude that there are so many unknowns that the set of physical parameters capable of reproducing the observed intensities cannot be uniquely determined by the $\mathrm{H}$ and $\mathrm{O} v \mathrm{v}$ line intensities and it becomes crucial to make good choices of the unknown parameters to reach a realistic solution.

To avoid biasing the results with a priori unjustified assumptions about the properties of the area at the edge of AR 8124, we assumed that the whole region we examined behaves like a typical streamer region. In this "whole streamer" hypothesis, we referred to results obtained by other authors to choose the unknown parameters of our simulation: we may expect a breakdown of our hypothesis at the edge of the AR, should outflows originate there, and in the southernmost pixels of the slit, where we are likely about to enter a polar coronal hole.

In streamer regions electron and kinetic temperatures are easily found in the literature: for the electron temperature we adopted the profile given by Gibson et al. (1999) and for the $\mathrm{O}_{\mathrm{VI}}$ kinetic temperatures we adopted those of Strachan et al. (2004). Both parameters change with altitude, with kinetic temperatures ranging from $2.0 \times 10^{6} \mathrm{~K}$ to $3.5 \times 10^{6} \mathrm{~K}$, over the 1.5 to $2.5 R_{\odot}$ altitude interval. UVCS observations of solar minimum streamers support the conclusion that kinetic perpendicular temperatures are higher than parallel temperatures above $2.6 R_{\odot}$ (Frazin et al. 2003). Since most of our data refer to lower levels, in this simulation we assumed an anisotropy ratio $D=T_{\| \mathrm{O} \text { VI }} / T_{\perp \mathrm{O} \text { VI }}=1$.

The Ly $\alpha$ kinetic temperatures were evaluated from our own data after correcting the line widths of the $\mathrm{H}_{\text {I }} \mathrm{Ly} \alpha$ for the instrumental profile function and the spectrometer line width (see Kohl et al. 1999). The kinetic temperature was $2 \times 10^{6} \mathrm{~K}$, increasing at the southernmost slit pixels to $3-4 \times 10^{6} \mathrm{~K}$ : this value compares 
well with the $3.5 \times 10^{6} \mathrm{~K}$ given by Cranmer et al. (1999) at an altitude of $2.5 R_{\odot}$ in a polar hole.

Most of the streamer plasma is quasi-static. Outflows were detected above the streamer cusp (see, e.g., Strachan et al. 2002), while Noci \& Gavryuseva (2007) claim to have found evidence of outflows of about $20 \mathrm{~km} \mathrm{~s}^{-1}$ throughout the body of the streamer. Spadaro et al. (2007) and Susino et al. (2008) claimed that oxygen ions move faster than hydrogen, in streamers above 3 solar radii and have negligible speeds at lower altitudes. Our data refer to lower altitudes, hence we assume in the following that proton and $\mathrm{O}$ vi ions move at the same speed. This hypothesis, as we will shortly see, allows us to leave the oxygen abundance as a free parameter. On the other hand, it will be difficult to assign a priori values to the oxygen abundances because they are known with substantial uncertainties and easily vary by one order of magnitude across a streamer (see, e.g. Raymond et al. 1997; Vásquez \& Raymond 2005); additionally, they decrease with height within these structures (see, e.g., Ko et al. 2002). We also expect them to change in an open field region, as shown by the different abundances in the slow and fast wind, which originate from differences in the respective source regions.

We adopted for the $\mathrm{H}_{\mathrm{I}} \mathrm{Ly} \alpha$ disk intensity the value given by the SOLSTICE (Solar Stellar Irradiance Comparison Experiment) on the UARS satellite (see e.g. Woods et al. 2000). Unfortunately, there are no measurement of the disk intensity in the O VI and C II lines in January 1998 and we had to resort to values given in the literature, which had been measured at disk center at solar minimum (Teriaca et al. 2003). However, we took into account that the $\mathrm{O}$ vi doublet lines have a marked limb brightening (see e.g. Withbroe 1970; Mariska et al. 1978) and that the chromospheric emissivity is not uniform across the disk. For the limb brightening, we mimicked the behavior of the $\mathrm{O}$ vi emission by using an analytic expression of the line intensities as a function of the angular distance from the disk center, which reproduces the steep brightness increase close to the limb and the smoother off-limb decline with the boundary condition of reproducing the measured values at the Sun center. We adopted the active region vs. quiet Sun contrast given by Vernazza \& Reeves (1978) for active regions across the disk, and modified the disk intensity by taking into account the area covered by active regions at the time of our data.

We point out that a $\pm 15 \%$ error in the assumed $\mathrm{O}$ vi and $\mathrm{C}_{\text {II }}$ disk brightness results in only $\pm 3 \%$ variations in the calculated values of outflows. Hence our choice is not crucial for evaluating the outflow speeds.

Once the electron temperature, the kinetic parallel and perpendicular temperatures and the disk intensities are known, as we said we furthermore assumed that protons and Ovi ions move at the same speed. There remained the unknown values of densities, outflow speeds and oxygen abundance, which we determined by reproducing the observed line intensities.

\subsubsection{The outflow speeds}

Outflow speeds from the simulation are given in Fig. 6a for the whole region of interest. Isocontours in Fig. 6a, show a very interesting behavior: in the region north of pixel 13 which (see Fig. 4), covers the streamer, there are virtually no outflows below 1.8 solar radii. However, a local outflow speed maximum is detected at pixels $13-14$ (corresponding to a dip in the isocontour map), where the $80-100 \mathrm{~km} \mathrm{~s}^{-1}$ speed, detected around 2.1 solar radii in equatorward pixels, is visible somewhat above the 1.5 solar radii level. Beyond pixel 14, isocontours
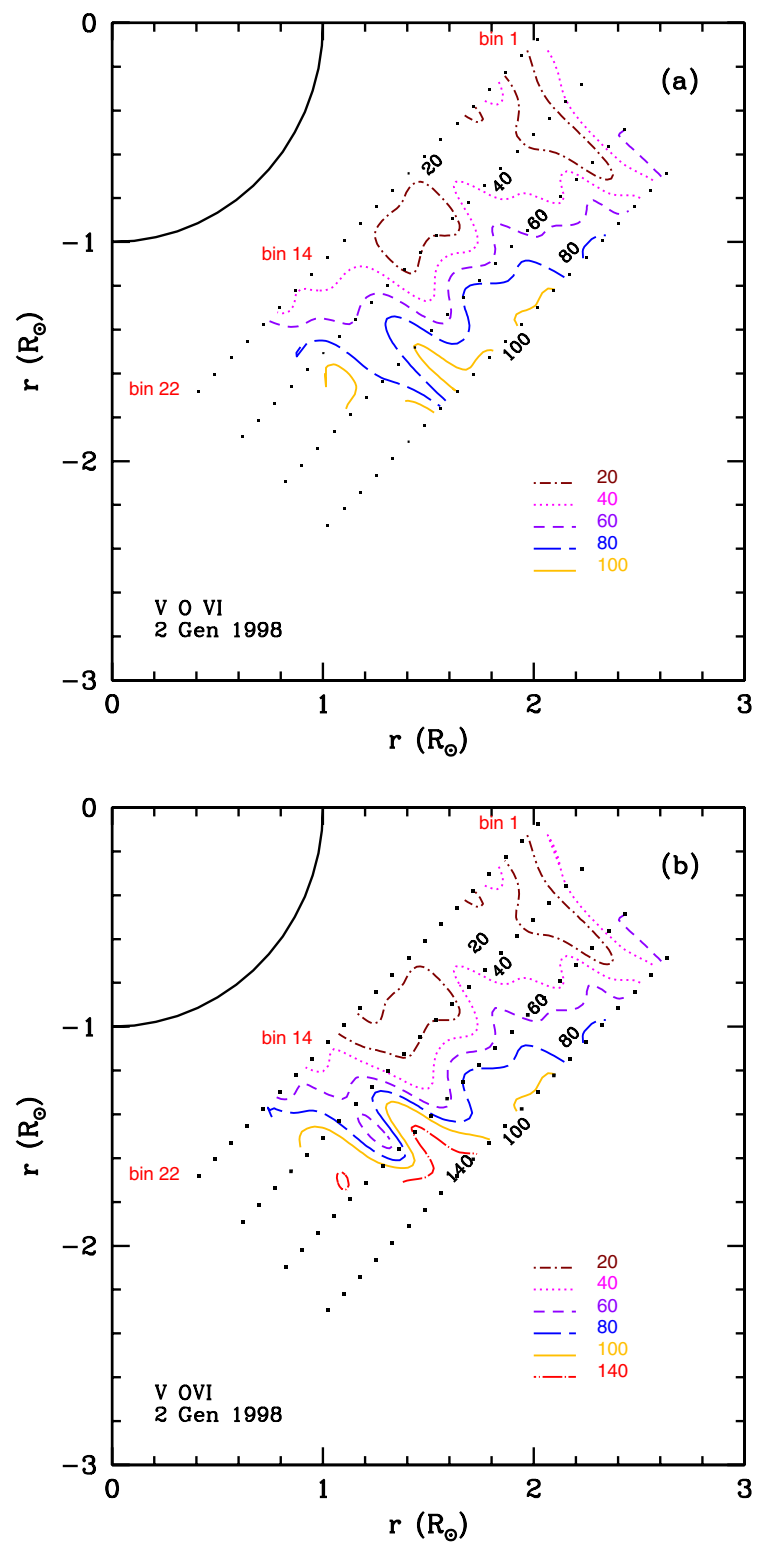

Fig. 6. Isocontour maps of $\mathrm{O}$ vi outflow speed $\left(\mathrm{km} \mathrm{s}^{-1}\right)$ in the $\mathrm{SW} \mathrm{sec-}$ tor of the corona. Dots along the slits centered at 1.5, 1.8, 2., 2.3 solar radii represent data points. Panel a) results from the "whole streamer" hypothesis; panel b) results assuming coronal hole parameters from bin 13 southward.

shift toward their pre-lane positions, before sinking downward when entering the coronal hole area. The lane of enhanced speed, where the plasma reaches at each heliocentric distance a higher speed than adjacent plasma, is located at 45 degree latitude, where potential field simulations show a bundle of open fieldlines originating at the edge of closed field structures within the AR. We conclude that the simulation suggests the occurrence of a lane, at $\approx 45$ degree latitude, where plasma is preferentially accelerated: this location corresponds to what we would expect if the AR boundaries were sources of solar wind.

The result we obtained from the simulation is encouraging, although we know that if some wind plasma is being accelerated at the AR edge, the hypotheses we made, which are appropriate for streamer plasmas, are likely to fail. We have some indications that this "whole streamer" hypothesis is not tenable at some locations: this occurs for slit 3 from bin 17 southward when the iterative procedure fails to find solutions, even for relatively 


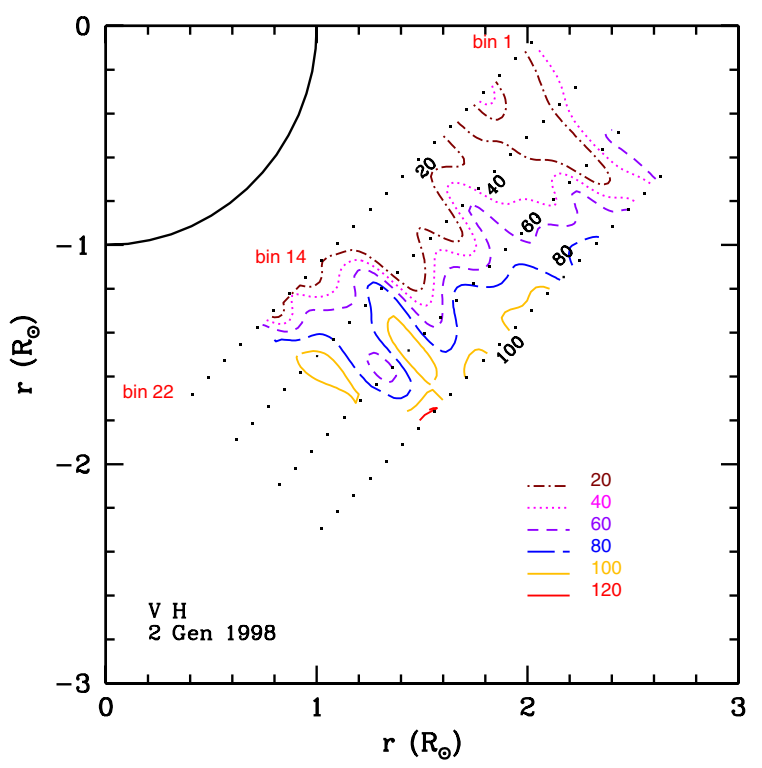

Fig. 7. Proton outflow speed $\left(\mathrm{km} \mathrm{s}^{-1}\right)$ isocontour map, deduced in coronal hole conditions starting from bin 13 outward.

high oxygen abundance values, suggesting that the hypothesis of equal outflow speeds for oxygen and protons is not acceptable. For slit 4, this lack of convergence starts occurring at bin 15.

As a consequence, on the basis of the outcome from the previous simulation, we conducted another search for the plasma parameters in the lane by assuming from pixel 13 onward that we are in the presence of a coronal hole plasma. Hence, we released the assumption for a unique outward speed for protons and $\mathrm{O} v \mathrm{vi}$ ions and looked for different speeds of the two species.

We assumed for the other parameters the coronal hole electron temperature profile that was adopted by Cranmer et al. (1999) in their coronal hole modeling and the kinetic temperatures of $\mathrm{O}$ VI ions from their model B1. For the temperature anisotropy we chose a ratio $D=T_{\| \mathrm{O} \text { VI }} / T_{\perp \mathrm{O} \text { VI }}=0.1$ and kept it constant with altitude. This is well within the allowed values of the anisotropy ratio in coronal holes, which according to Cranmer (2002), reach values from 0.1 to 0.01 at heights from 2 solar radii onward.

If protons and $\mathrm{O}_{\mathrm{VI}}$ ions move at different speeds, we have an additional unknown quantity and we need to fix the value of some other parameter. In the previous simulation, oxygen abundance had been left as a free parameter and the values we obtained were ranging in the body of the streamer between 8.1 and 8.5, which agrees well with other determination (see e.g. Marocchi et al. 2001; Parenti et al. 2000). Values of oxygen abundance are given as $\log \left(n_{\text {oxy }} / n_{\mathrm{H}}\right)+12$, where $n_{\text {oxy }}$ and $n_{\mathrm{H}}$ are the oxygen and hydrogen numerical densities, respectively. In coronal holes, however, the oxygen abundance is higher and we set its value to 8.7 in agreement with findings for coronal hole (e.g. Zangrilli et al. 2002), photospheric (Allende Prieto et al. 2001) and fast wind (von Steiger et al. 1999) abundances; all of which should be consistent if coronal holes are sources of fast wind. Results from this simulation are shown in Fig. 6b. The higher outward speed lane appears in this simulation as well, with enhanced values of the speed with respect to the previous model. O vi ions move outward at a speed of $\approx 140 \mathrm{~km} \mathrm{~s}^{-1}$ at an altitude of 2 solar radii. The proton outflow speeds deduced in typical coronal hole conditions are shown in Fig. 7, where profiles similar to the $\mathrm{O}$ vi ones are obtained, although generally attaining lower values.
Table 1. Error affecting the speed at bin 14.

\begin{tabular}{lccc}
\hline \hline $\begin{array}{l}\text { Height } \\
\left(R_{\odot}\right)\end{array}$ & $\begin{array}{c}v \\
\left(\mathrm{~km} \mathrm{~s}^{-1}\right)\end{array}$ & $\begin{array}{c}v-\Delta v \\
\left(\mathrm{~km} \mathrm{~s}^{-1}\right)\end{array}$ & $\begin{array}{c}v+\Delta v \\
\left(\mathrm{~km} \mathrm{~s}^{-1}\right)\end{array}$ \\
\hline Whole streamer & & & \\
1.5 & 20 & 0 & 41 \\
1.8 & 65 & 50 & 77 \\
2.1 & 101 & 90 & 111 \\
\hline Coronal hole & & & \\
1.5 & 35 & 0 & 59 \\
1.8 & 73 & 52 & 91 \\
2.1 & 143 & 114 & 178 \\
\hline
\end{tabular}

Notes. The estimate was obtained considering the maximum and minimum values of the $\mathrm{O}$ vi doublet intensity ratio.

Hence, both simulations, representative of plasma in extremely different conditions, agree and support a scenario where an high outward flow occurs along open fieldlines at the edge of AR 8124. Our simulations show in this lane O vI outflows reaching about $150 \mathrm{~km} \mathrm{~s}^{-1}$ at $\approx 2$ solar radii, and protons flowing at an $\approx 30 \%$ lower speed.

To estimate the uncertainties in the values inferred for outflows, we estimated the upper limit of the error that affects the value of the speed at bin 14 in the high speed lane throughout the first three slits. To this end we changed the O vi doublet line intensities assuming that the 1031.9 and 1037.6 lines had reached their maximum and minimum values, considering the uncertainties given by the Poissonian statistics. In this way, we set the 1031.9/1037.6 line ratio to its maximum value. We then made the opposite assumption, setting the 1031.9 and 1037.6 lines to their minimum and maximum intensity, and obtained the minimum value for the $1031.9 / 1037.6$ ratio. The speed at bin 14 is affected by the maximum error shown in Table 1 .

We furthermore notice that a different choice of the oxygen abundance will affect the electron density determination, but the oxygen outflow speed value will hardly change. This is because the outflow speed depends on the line ratio, not on the assumed ion abundance.

\subsubsection{Electron density}

Another check of the reliability of our results can be made by analyzing the profile of the electron density in the outflowing lane. This is shown in Fig. 8, where electron densities in streamers (Gibson et al. 1999) and coronal holes (Guhathakurta et al. 1999) are given as well. Figure 8 shows that independent of whatever simulation is selected, densities in the lane are higher than coronal hole densities and lower than typical streamer conditions. They appear to be representative of an intermediate condition between the low-density coronal hole plasma and the high streamer density that might be expected from plasma at the AR boundary. We also calculated the electron density values along the streamer axis (see triangles in Fig. 8), which are consistent with the radial electron density profile given by Gibson et al. (1999).

In coronal regions where no relevant outflow is inferred from the above Doppler dimming analysis, the calculated electron densities can also be compared with those obtained from the ratio of the collisional to the radiative component of the O vi $1031.9 \AA$ line intensity, as suggested by Noci et al. (1987) (see also Parenti et al. 2000; Ko et al. 2006):

$\frac{I_{\text {res }}}{I_{\text {coll }}}=5.75 \times 10^{2} \frac{\lambda^{2} \exp (E / k T) \sqrt{T} \int I_{\text {disk }}(\lambda) \mathrm{d} \lambda}{\bar{g} n_{\mathrm{e}}\left(\Delta \lambda_{\text {cor }}^{2}+\Delta \lambda_{\mathrm{ex}}^{2}\right)^{1 / 2}}\left(\frac{R_{\odot}}{r}\right)^{2} Q(r)$, 


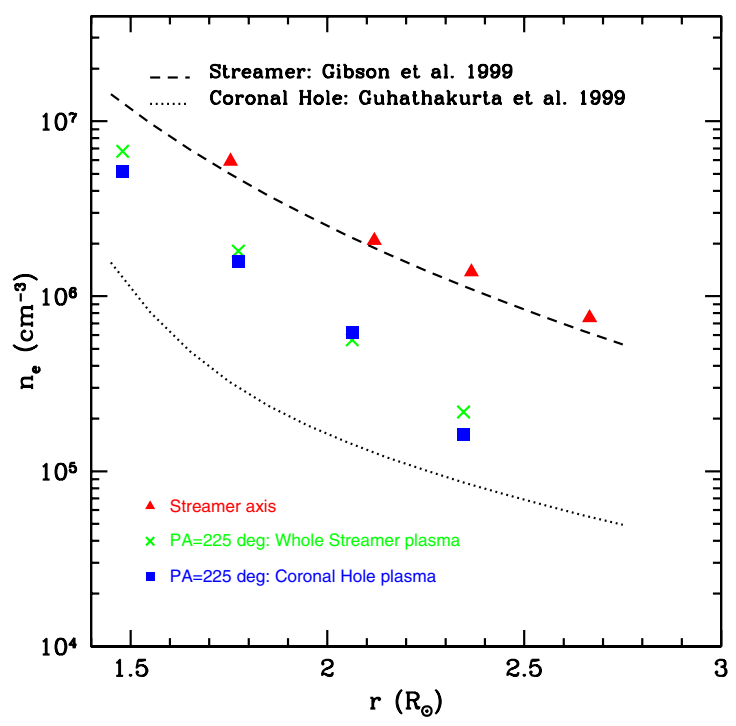

Fig. 8. Electron densities derived from the Doppler dimming analysis compared with profiles from literature relative to the typical coronal hole and streamer.

where $\lambda=1031.9 \AA, E=1.9251 \times 10^{-11}$ erg is the transition energy, $T$ is the electron temperature, $I_{\text {disk }}$ is the line intensity integrated over the disk, $\bar{g}=1.13$ is the effective Gaunt factor, $\Delta \lambda_{\mathrm{ex}}=0.10 \AA$ is the $1 / e$ half-width of the exciting line from the lower atmosphere, $\Delta \lambda_{\text {cor }}=0.11 \AA$ is the $1 / e$ half-width of the coronal absorption profile, $Q(r)=2\left[1-\left(1-R_{\odot}^{2} / r^{2}\right)^{1 / 2}\right] r^{2} / R_{\odot}^{2}$ is the solid angle subtended by the solar disk at a distance $r$, and $n_{\mathrm{e}}$ is the electron density. For more details see Noci et al. (1987). This technique is based on the $n_{\mathrm{e}}^{2}$ dependence of the collisional vs. the $n_{\mathrm{e}}$ dependence of the radiative line component and can be applied only in nearly static plasma conditions. We point out that the radiative to collisional intensity ratio is independent of the assumed oxygen abundance, and that the two components can be easily separated with the two conditions of Eq. (1), when $\mathcal{P}=0$. It turns out that the electron densities derived with the two methods agree within $10 \%$, supporting the validity of our approach also in a nearly static plasma regime.

There is yet another independent method to determine the coronal electron density from the inversion of the $\mathrm{pB}$ data, acquired by coronagraphs (see van de Hulst 1950). The Mk3 coronagraph at Mauna Loa has no data on the day of our observations, the closest time being the 31 December 1997, when part of the coronal region of our interest was observed. Polarized brightness data were inverted with the pBinverter procedure, provided by the SolarSoft package. A good agreement was obtained along radial directions close to the equator, with our values being some 15-20\% higher than results from the $\mathrm{pB}$ inversion.

We conclude that estimates made by the procedure described in Sect. 3 agree with those obtained with $\mathrm{pB}$ inversion and with the collisional to radiative ratio of the $1031.9 \AA$ line components, and nicely fit what can be expected in different regions from values given in the literature. This supports our confidence in the results presented here. Nevertheless, we proceeded to make another check of the reliability of the values we inferred for plasma outflows.

\subsubsection{Calculation of $H I L y \beta$ intensity}

In Sect. 3.1.3 we showed that the density values we derived are realistic: however, we did not directly check the reliability

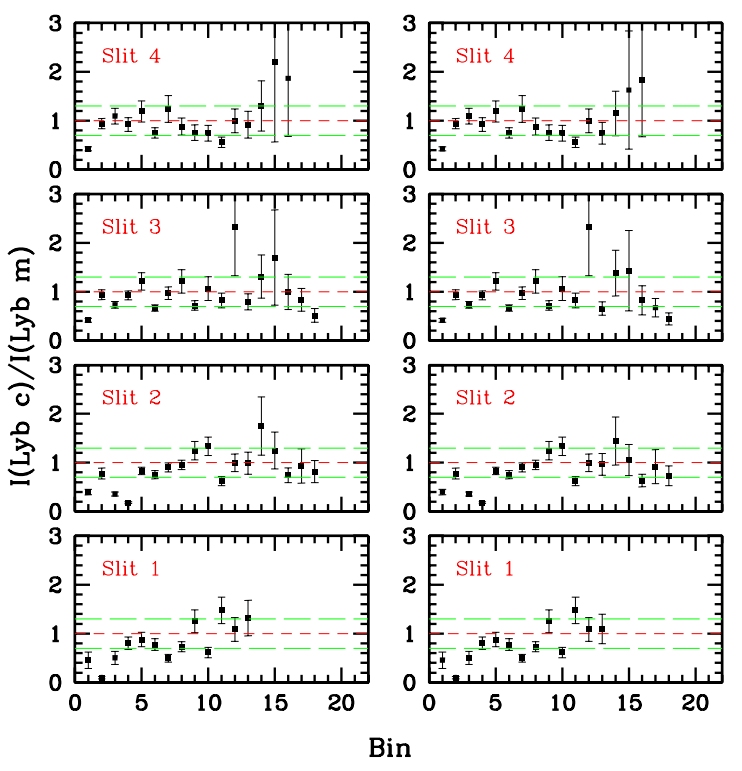

Fig. 9. Ratio of predicted over observed $\mathrm{H}_{\mathrm{I}} \mathrm{Ly} \beta$ intensities, $\left.\left.I_{\mathrm{Ly} \beta, \mathrm{c}}\right) / I_{\mathrm{Ly} \beta, \mathrm{m}}\right)$, in the whole streamer case (left panels) and in the coronal hole plasma conditions (right panels).

of the outflow values. To this end, we computed the intensities of the $\mathrm{H}_{\text {I }} \mathrm{Ly} \beta$ line on the basis of the physical parameters we inferred, and compared the predicted and observed values. Because the $\mathrm{H}_{\text {I }} \mathrm{Ly} \beta$ has both a collisional and a radiative component, which of course is affected by the outflow plasma motion, this comparison is a more direct check of our results.

The predicted/observed intensity ratio, $I_{\mathrm{Ly} \beta, \mathrm{c}} / I_{\mathrm{Ly} \beta, \mathrm{m}}$, is shown in Fig. 9; error bars were calculated from Poissonian statistics: because this is a faint line, uncertainties are larger than for

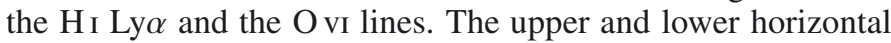
dashed lines delimitate the region in which the calculated and measured intensities differ by less than $30 \%$. Most of the times the $\mathrm{H}_{\mathrm{I}} \mathrm{Ly} \beta$ intensities are reproduced within $30 \%$, which is fairly acceptable because of the uncertainties that affect the measured intensities.

\section{Discussion and conclusions}

We have revealed, for the first time, the occurrence of coronal outflows at the edge of active regions, between 1.5 and 2.3 solar radii, via a Doppler dimming analysis of data acquired by SOHO/UVCS on January 2, 1998, when AR 8124 was at the west limb of the Sun. The outflow was confined within a narrow stream lane, adjacent to closed loop systems, at a location at which potential field extrapolations show a bundle of open magnetic field lines. As we mentioned in the introduction, while there are many works that described/measured this kind of outflows from data acquired at low coronal altitudes, only a few works provided indirect evidence for the persistence of outflows at higher levels. Among those, we cited the paper by Ko et al. (2006) who, from an UVCS coronal and ACE in situ abundance analysis, supported the idea of a slow wind source at the boundary between ARs and CHs. An analogous work has been performed recently by Brooks \& Warren (2011), who compared the chemical composition of an AR observed by HINODE/EIS over a few days with the ACE/SWICS composition and found a general agreement between the two abundances.

A different approach has been taken by Del Zanna et al. (2011) and Bradshaw et al. (2011), who found indirect evidence 


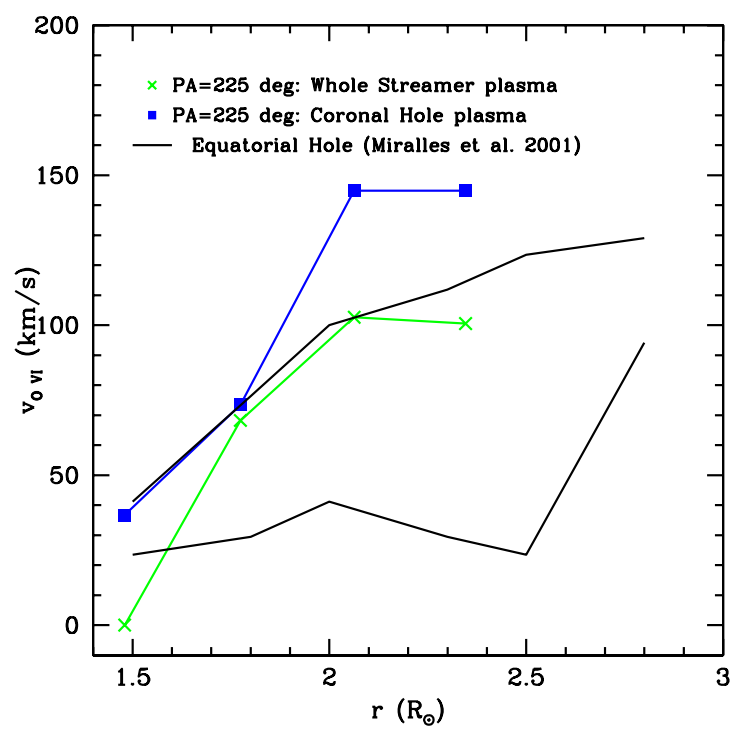

Fig. 10. Outflow speeds derived along the radial at $225 \mathrm{deg}$, in the highspeed lane we located, and the outflows inferred by Miralles et al. (2001) in an equatorial coronal hole. The continuous black lines give the upper and lower limit for the outflow speed in the equatorial hole.

for the persistence at higher coronal heights of the outflows detected in the low corona by HINODE/EIS in the radio noise storms, observed with the Nancay radioheliograph, which map electron beams propagating in the high corona. According to these authors, interchange reconnection between active region loops and nearby lower density loops triggers the formation of new (closed and) open fieldlines where a field-aligned pressure gradient accelerates upflowing plasma and low-energy electrons, leading to the observed noise storms. The fraction of plasma that can be injected into the interplanetary space as a consequence of this process is not specified but may be a source of solar wind inhomogeneities.

In contrast to previous works, this analysis provided direct evidence of coronal outflows, although we are unable to uniquely identify the height vs. outward speed profile of the AR plasma because we lack a direct measurement of several parameters. However, we show in Fig. 10 a comparison of the speed vs. altitude profile we inferred with typical slow-wind profiles from equatorial holes (Miralles et al. 2001), in which we see that independent of whatever choice of the parameters we make (i.e. streamer or $\mathrm{CH}$ simulation), above 1.8 solar radii AR outflows are larger than $\mathrm{CH}$ outflows. Measurements of the oxygen abundance in the low corona, at the root of the flows, and of the kinetic temperature of oxygen ions at high coronal altitudes may help to narrow the choices of the free parameters and lead to a better defined speed vs. altitude profile.

Our analysis yielded no results for the intermittency vs. persistence of AR flows. Harra et al. (2008) and Sakao et al. (2007), for instance, observed persistent outflows at the edge of ARs, while He et al. (2010) and Guo et al. (2010) found intermittent and quasi-periodic flows at AR boundaries, respectively. Original data have an integration time of $100 \mathrm{~s}$ : at each slit position, however, data were integrated over 300, 500, 700, $900 \mathrm{~s}-$ i.e. over the total observing time at each altitude at which the slit was set - to obtain a better statistics. Because He et al. (2010) gave a value of $20 \mathrm{~min}$ as a typical time interval between outflows episodes, and Guo et al. (2010) gave 5-10 min as typical flow periodicities, our data do not allow us to check the intermittency/periodicity question.
Densities in the AR outflowing plasma are higher than typical polar coronal hole densities. Measurements of densities in equatorial holes in the high corona at 1.5-2.5 solar radii altitude interval are missing, but we may hypothesize that the 2.7 density enhancement factor of equatorial over polar holes given by Gallagher et al. (1999) applies also at this altitude interval. We may then compare, for a flux tube of the same section, the mass flux from an AR outflow, calculated from average speed and densities at 2 solar radii, to the mass flux at the same level of a similar flux tube in coronal holes. The mass flux is higher in AR flux tubes than in equatorial and polar holes by roughly a factor of 2.1 and 1.6, respectively. This may support the estimate of a substantial contribution of ARs to the wind mass flux made by several authors (e.g. Sakao et al. 2007) although for a precise calculation of this contribution more information are needed.

Acknowledgements. The pB Mk3 data used in this work have been obtained as courtesy of the Mauna Loa Solar Observatory, operated by the High Altitude Observatory, as part of the National Center for Atmospheric Research (NCAR). NCAR is supported by the National Science Foundation. G.P. acknowledges support from ASI I/023/09/0.

\section{Appendix A: Collisional and radiative components of the $\mathrm{O} \mathrm{VI}$ doublet lines}

The collisional and radiative components of the coronal O vi doublet lines depend on the density and outflow plasma speed. However, they depend also, although more weakly, on other parameters that can be assigned a priori, such as the coronal electron temperature, the $\mathrm{O}$ vi disk chromospheric intensity and the atomic line parameters. More precisely, the intensity of the $\mathrm{O}$ vi lines is given by

$I=\int_{-\infty}^{+\infty}\left(j_{\text {coll }}+j_{\text {res }}\right) \mathrm{d} x=C+\mathcal{R}+\mathcal{P}$,

where $j_{\text {coll }}$ and $j_{\text {res }}$ are the collisional and radiative emissivities, respectively, and the integration is made along the $\operatorname{LOS}(\mathcal{P}=0$ for the $1031.9 \AA$ line). Terms $C, \mathcal{R}$ and $\mathcal{P}$ are defined as in Eq. (1).

The collisional emissivity for the Earth observer at a point $\mathrm{P}$ at the frequency $v$ can be expressed in units of $\mathrm{erg} / \mathrm{s} / \mathrm{cm}^{3} / \mathrm{sr} / \AA$ as

$j_{\text {coll }}(P, v)=\frac{h v_{0}}{4 \pi} n\left(X^{+m}\right) n_{\mathrm{e}} C_{\text {coll }} \phi_{\mathrm{c}}(v)$,

where $v_{0}$ is the frequency of the transition, $n\left(X^{+m}\right)$ is the ground state number density of the $m$-times ionized element $X, n_{\mathrm{e}}$ is the electron density, $C_{\text {coll }}$ is the collisional coefficient of the transition, $\phi_{\mathrm{c}}$ is the coronal emission profile. Usually, ionization equilibrium is assumed, and

$$
\begin{aligned}
n\left(X^{+m}\right) & =\frac{n\left(X^{+m}\right)}{n(X)} \frac{n(X)}{n_{\mathrm{H}}} \frac{n_{\mathrm{H}}}{n_{\mathrm{e}}} n_{\mathrm{e}} \\
& =R_{\mathrm{ion}} A_{\mathrm{el}} 0.83 n_{\mathrm{e}},
\end{aligned}
$$

where $A_{\mathrm{el}}$ is the element abundance and a plasma composition with $10 \%$ of helium has been assumed.

Adopting now a cartesian system with the origin in a point $P$, at a distance $\boldsymbol{r}$ from the Sun center, the $X$ axis along $\boldsymbol{n}$ (direction toward the observer) and the $Z$ axis in the plane $\boldsymbol{n}, \boldsymbol{r}$ (directed toward the Sun), we can define the distribution function 
of the speed of the absorbing ions as $f\left(v_{\mathrm{X}}(v), v_{\mathrm{Y}}, v_{\mathrm{Z}}\right)$ and write the expression for the emissivity of the radiative component as

$$
\begin{aligned}
j_{\text {res }}(P, v, \boldsymbol{n})= & \frac{n\left(X^{+m}\right) B_{12} h v_{0}}{4 \pi} \int_{\Omega_{\odot}} p\left(\boldsymbol{n} \cdot \boldsymbol{n}^{\prime}\right) \mathrm{d} \omega^{\prime} \\
& \times \int_{-\infty}^{+\infty} I_{\mathrm{D}}\left(v^{\prime}\left[v_{\mathrm{X}}(v), v_{\mathrm{Y}}, v_{\mathrm{Z}}, v_{0}, \boldsymbol{n}^{\prime}\right]\right) \\
& \times f\left(v_{\mathrm{X}}(v), v_{\mathrm{Y}}, v_{\mathrm{Z}}\right) \mathrm{d} v_{\mathrm{Y}} \mathrm{d} v_{\mathrm{Z}},
\end{aligned}
$$

where $\boldsymbol{n}^{\prime}$ is the direction of propagation of the radiation coming from the disk, $B_{12}$ is the Einstein coefficient of absorption, $p$ is the angular dependence function of the scattering process, $n\left(X^{+m}\right)$ the number density of absorbing ions, and $v^{\prime}$ is the frequency of the absorbed photon, related to the transition frequency by $v^{\prime}=v_{0}\left(1+v_{n^{\prime}} / c\right)$, where $v_{n^{\prime}}=\boldsymbol{v} \cdot \boldsymbol{n}^{\prime}$. The disk intensity is $I_{\mathrm{D}}$, and $\Omega_{\odot}$ is the solid angle subtended by the solar disk. The radiative emissivity is a function of the local plasma outflow speed $\boldsymbol{v}$ via the Doppler dimming effect. The speed $\boldsymbol{v}$ of an absorbing ion can be considered as the sum of its random microscopic speed (assuming that the plasma is in thermal equilibrium) and the outflow speed of the wind.

The speed distribution function is often described in terms of a bi-maxwellian function:

$$
f(\boldsymbol{v})=\left(\frac{m}{2 \pi k T_{\|}}\right)^{1 / 2} \frac{m}{2 \pi k T_{\perp}} \exp \left[-\frac{m}{2 k T_{\|}}\left(v_{\|}-w\right)^{2}-\frac{m}{2 k T_{\perp}} v_{\perp}^{2}\right],
$$

where $w$ is the outflow wind speed, $v_{\|}$and $v_{\perp}$ are the thermal speed parallel and perpendicular to the magnetic field lines, respectively, and $T_{\|}, T_{\perp}$ are the kinetic temperatures along the same directions. Anisotropic temperature distributions are needed to model line intensities in coronal holes and also to account for the broad line widths of the Ovi lines observed in those regions. The anisotropy appears above $\approx 2$ solar radii and takes values of the ratio $D=T_{\| \mathrm{O} \text { VI }} / T_{\perp \mathrm{O} \text { VI }}$ of about 0.1 to 0.01 (Cranmer 2002; Cranmer et al. 2008) in CHs. Note that the anisotropy in Cranmer (2002) is defined as $1 / D$. In streamer regions there is, if any, a much lower anisotropy (Frazin et al. 2003).

\section{References}

Allende Prieto, C., Lambert, D. L., \& Asplund, M. 2001, ApJ, 556, L63 Bradshaw, S. J., Aulanier, G., \& Del Zanna, G. 2011, ApJ, 743, 66 Brooks, D. H., \& Warren, H. P. 2011, ApJ, 727, L13

Cranmer, S. R. 2002, in From Solar Min to Max: Half a Solar Cycle with SOHO, ed. A. Wilson, ESA SP, 508, 361
Cranmer, S. R., Kohl, J. L., Noci, G., et al. 1999, ApJ, 511, 481

Cranmer, S. R., Panasyuk, A. V., \& Kohl, J. L. 2008, ApJ, 678, 1480 Cranmer, S. R., Gardner, L. D., \& Kohl, J. L. 2010, Sol. Phys., 263, 275 Del Zanna, G., Aulanier, G., Klein, K.-L., \& Török, T. 2011, A\&A, 526, A137 Domingo, V., Fleck, B., \& Poland, A. I. 1995, Sol. Phys., 162, 1 Doschek, G. A., Warren, H. P., Mariska, J. T., et al. 2008, ApJ, 686, 1362 Frazin, R. A., Cranmer, S. R., \& Kohl, J. L. 2003, ApJ, 597, 1145 Gallagher, P. T., Mathioudakis, M., Keenan, F. P., Phillips, K. J. H., \& Tsinganos, K. 1999, ApJ, 524, L133

Gibson, S. E., Fludra, A., Bagenal, F., et al. 1999, J. Geophys. Res., 104, 9691 Green, L. M., Harra, L. K., Matthews, S. A., \& Culhane, J. L. 2001, Sol. Phys., 200, 189

Green, L. M., López fuentes, M. C., Mandrini, C. H., et al. 2002, Sol. Phys., 208, 43

Guhathakurta, M., Fludra, A., Gibson, S. E., Biesecker, D., \& Fisher, R. 1999, J. Geophys. Res., 104, 9801

Guo, L.-J., Tian, H., \& He, J.-S. 2010, Res. Astron. Astrophys., 10, 1307 Harra, L. K., Sakao, T., Mandrini, C. H., et al. 2008, ApJ, 676, L147 He, J.-S., Marsch, E., Tu, C.-Y., Guo, L.-J., \& Tian, H. 2010, A\&A, 516, A14 Kamio, S., Peter, H., Curdt, W., \& Solanki, S. K. 2011, A\&A, 532, A96 Ko, Y.-K., Raymond, J. C., Li, J., et al. 2002, ApJ, 578, 979 Ko, Y.-K., Raymond, J. C., Zurbuchen, T. H., et al. 2006, ApJ, 646, 1275 Kohl, J. L., Esser, R., Gardner, L. D., et al. 1995, Sol. Phys., 162, 313 Kohl, J. L., Noci, G., Antonucci, E., et al. 1997, Sol. Phys., 175, 613 Kohl, J. L., Esser, R., Cranmer, S. R., et al. 1999, ApJ, 510, L59 Kojima, M., Fujiki, K., Ohmi, T., et al. 1999, J. Geophys. Res., 104, 16993 Krieger, A. S., Timothy, A. F., \& Roelof, E. C. 1973, Sol. Phys., 29, 505 Liewer, P. C., Neugebauer, M., \& Zurbuchen, T. 2004, Sol. Phys., 223, 209 Mariska, J. T., Feldman, U., \& Doschek, G. A. 1978, ApJ, 226, 698 Marocchi, D., Antonucci, E., \& Giordano, S. 2001, Ann. Geophys., 19, 135 Miralles, M. P., Cranmer, S. R., Panasyuk, A. V., Romoli, M., \& Kohl, J. L. 2001, ApJ, 549, L257

Noci, G., \& Gavryuseva, E. 2007, ApJ, 658, L63

Noci, G., Kohl, J. L., \& Withbroe, G. L. 1987, ApJ, 315, 706

Parenti, S., Bromage, B. J. I., Poletto, G., et al. 2000, A\&A, 363, 800

Raymond, J. C., Kohl, J. L., Noci, G., et al. 1997, Sol. Phys., 175, 645

Sakao, T., Kano, R., Narukage, N., et al. 2007, Science, 318, 1585

Spadaro, D., Susino, R., Ventura, R., Vourlidas, A., \& Landi, E. 2007, A\&A, 475,707

Strachan, L., Suleiman, R., Panasyuk, A. V., Biesecker, D. A., \& Kohl, J. L. 2002, ApJ, 571, 1008

Strachan, L., Baham, M., Miralles, M. P., \& Panasyuk, A. V. 2004, in SOHO 15 Coronal Heating, eds. R. W. Walsh, J. Ireland, D. Danesy, \& B. Fleck, ESA SP, 575, 148

Susino, R., Ventura, R., Spadaro, D., Vourlidas, A., \& Landi, E. 2008, A\&A, 488, 303

Teriaca, L., Poletto, G., Romoli, M., \& Biesecker, D. A. 2003, ApJ, 588, 566

Ugarte-Urra, I., \& Warren, H. P. 2011, ApJ, 730, 37

van de Hulst, H. C. 1950, Bull. Astron. Inst. Netherlands, 11, 135

Vásquez, A. M., \& Raymond, J. C. 2005, ApJ, 619, 1132

Vernazza, J. E., \& Reeves, E. M. 1978, ApJS, 37, 485

von Steiger, R., Fisk, L. A., Gloeckler, G., Schwadron, N. A., \& Zurbuchen, T. H. 1999, in AIP Conf. Ser. 471, eds. S. R. Habbal, R. Esser, J. V. Hollweg, \& P. A. Isenberg, 143

Winebarger, A. R., DeLuca, E. E., \& Golub, L. 2001, ApJ, 553, L81

Withbroe, G. L. 1970, Sol. Phys., 11, 42

Woods, T. N., Tobiska, W. K., Rottman, G. J., \& Worden, J. R. 2000, J. Geophys. Res., 105, 27195

Zangrilli, L., Poletto, G., Nicolosi, P., Noci, G., \& Romoli, M. 2002, ApJ, 574, 477 